\title{
Nanoporous platinum electrode grown on anodic aluminum oxide membrane: Fabrication, characterization, electrocatalytic activity toward reactive oxygen and nitrogen species
}

\author{
Wenyan Tao ${ }^{\text {a, b}}$, Dawei Pan ${ }^{\text {c, d, }}{ }^{*}$, Zanhua Gong ${ }^{a}$, Xia Peng a \\ a Shenzhen Huachenyang Technology Company, Shenzhen, 510081, PR China \\ ${ }^{\mathrm{b}}$ Department of Electronic Engineering, The Chinese University of Hong Kong, Hong Kong, PR China \\ ${ }^{\mathrm{c}}$ Key Laboratory of Coastal Environmental Processes and Ecological Remediation, Yantai Institute of Coastal Zone Research, Chinese Academy of Sciences, \\ Yantai, 264003, PR China \\ ${ }^{\mathrm{d}}$ University of Chinese Academy of Sciences, Beijing, 100049, PR China
}

\section{H I G H L I G H T S}

- A new type of nanoporous platinum (NPt) electrode was fabricated on porous aluminum oxide membrane at the first time.

- Electrocatalytic mechanisms of NPt electrode toward NO and nitroxyl were proposed, which depended on $\mathrm{pH}$ value of buffer.

- NPt electrode exhibited three times larger catalytic current to $\mathrm{O}_{2}$, $\mathrm{NO}$ and nitroxyl than those on nanofilm $\mathrm{Pt}$ electrode.

\section{A R T I C L E I N F O}

\section{Article history:}

Received 20 April 2018

Received in revised form

14 June 2018

Accepted 29 June 2018

Available online 30 June 2018

\section{Keywords:}

Nanoporous platinum electrode

Nitric oxide

Angeli's salt

Thermal evaporation deposition

\section{G R A P H I C A L A B S T R A C T}

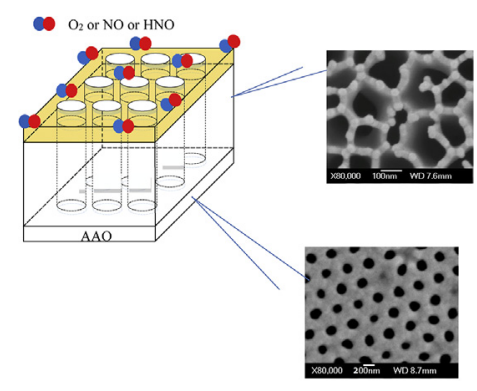

\begin{abstract}
A B S T R A C T
A new type of nanoelectrode, nanoporous platinum (NPt) electrode was prepared on aluminum oxide membrane by thermal evaporation deposition. The morphology, conductivity and electrocatalytic activity of NPt electrode were characterized and compared with those of nanofilm-Pt electrode through scanning electron microscopy (SEM), electrochemical impedance spectroscopy (EIS) and cyclic voltammetry (CV) techniques, respectively. SEM images showed that "nanocavities" observed in NPt electrode were actually 2-dimensional enclosures by linked nanoparticles. It was different from the conventional arrays of "nanocavities" formed on homogeneous metal films. EIS data indicated that NPt electrode possesses higher conductivity. Compared with that on nanofilm-Pt electrode $\left(14.05 \Omega \cdot \mathrm{cm}^{2}\right)$, the impedance spectrum on NPt electrode exhibits a semicircle portion with much smaller diameters (1.24 $\Omega \cdot \mathrm{cm}^{2}$ for NPt-100, $1.48 \Omega \cdot \mathrm{cm}^{2}$ for NPt-200). Meanwhile, the response sensitivity of NPt electrode to $\mathrm{O}_{2}$ is $0.85 \mathrm{~mA} \mathrm{~cm}^{-2}$, which is larger than that of nanofilm-Pt electrode $\left(0.54 \mathrm{~mA} \mathrm{~cm}^{-2}\right)$. The largest catalytic current for nitric oxide (NO) was obtained in buffer with pH value of 9.4 while for Angeli's salt (AS) was obtained in buffer with $\mathrm{pH}$ value of 5.4. Additionally, electrocatalytic mechanisms of NPt electrode toward NO and AS were proposed, which indicating it depended on $\mathrm{pH}$ value of buffer solution.
\end{abstract}

() 2018 Elsevier B.V. All rights reserved.

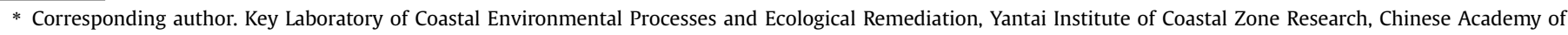
Sciences, Yantai, 264003, PR China.

E-mail address: dwpan@yic.ac.cn (D. Pan).
} 


\section{Introduction}

Platinum (Pt) nanoparticles have been received wide interests in various fields, such as biosensor [1], catalysis [2], fuel cell [3], optoelectronic device [4] and so on. Template synthesis is an effective method for preparing nanomaterial with defined dimension [5]. Present techniques to form nanomaterials on templates have been developed including chemical vapor deposition (CVD), sol-gel deposition, polymerization, and electrochemical deposition [6]. However, Pt nanomaterials for electro-catalysis application were depended on synergetic effect of other matrix, such as Nafion and carbon, which involves complicated fabrication process. On the other hand, porous anodized aluminum oxide (AAO) substrates have become feasible, low-cost, versatile inorganic materials for wide applications in filtration [7], catalysis [8], biosensing [9] and nanofilm-fabrication [10]. AAO substrates consist of highly-ordered and uniform nanopores with large depth/width ratio (>1000) [11]. The pore diameter and depth can be adjusted by the anodization conditions - acid types, $\mathrm{pH}$ value, temperature and anodizing time [12]. AAO is the typical template employed for nano-structural film fabrication. AAO provides a rough surface with large surface area for supporting metal nanoparticles and formed a conductive nanostructural network which facilitates measurement of electrical property. Until now, there is few work based on AAO template for Pt nanoparticle film-fabrication.

Reactive nitrogen species including oxide species-nitric oxide (NO) and reduced species - nitroxyl (HNO) in biological system have been known for many years. NO plays an important role in various physiological processes, serving as an endothelial-derived relaxing factor [13], a neurotransmitter [14], and an immune system mediator [15]. Electrocatalytic detection of NO was mainly operated in acidic medium, which limited the application $\mathrm{pH}$ range [16]. Therefore, it is significant to study electrocatalytic detection of NO in whole $\mathrm{pH}$ range cover from acidic to alkaline solution. Nitroxyl (HNO), a protonated one-electron reduction product of nitric oxide, is a transient species that has been implicated in the biological activity of nitric oxide. It is donated from Angeli's salt (AS):

$\mathrm{HN}_{2} \mathrm{O}_{3}^{-} \rightarrow \mathrm{HNO}+\mathrm{NO}_{2}^{-}$

In aqueous solution, it spontaneously dimerizes to give nitrous oxide, which results in its role in biology greatly neglected or dismissed:

$2 \mathrm{HNO} \rightarrow \mathrm{N}_{2} \mathrm{O}+\mathrm{H}_{2} \mathrm{O}$

However, many reports have been published to study of HNO biology in recent years. Cetraz [17] et al. investigated a newly synthesized platinum-nitroxyl complex for their potential to circumvent cisplatin resistance in RT112 bladder cancer cells. Recent progress has been made on the elucidation of the mechanisms associated with HNO biological signaling. Importantly, the biochemical mechanisms described for HNO bioactivity are consistent with its unique and novel chemical properties/reactivity [18]. Doctorovich et al. reported the reactions of HNO with metal porphrins. Kinetic and mechanistic studies of these reactions show two alternative operating mechanisms: reaction of the metal center with HNO or with the donor molecules such as trioxodinitrate (Angeli's salt) [19].

In this work, nanoporous Pt electrode fabricated on AAO substrate was used as a novel electrode for electro-catalysis of reactive nitrogen species-NO and HNO. Meanwhile, Pt nanoparticle was prepared on $\mathrm{N}$-type silicon wafer as a control system, named as
nanofilm-Pt electrode. Their morphology and electrochemical performances were studied by scanning electron microscopy (SEM), cyclic voltammetry (CV) and electrochemical impedance spectroscopy (EIS). The electro-catalysis of reactive oxygen $\left(\mathrm{O}_{2}\right)$ and nitrogen species (NO and HNO) on nanoporous Pt electrode are compared with those on nanofilm-Pt electrode.

\section{Experimental section}

\subsection{Reagent}

Porous anodic aluminum oxide (AAO) membrane with average pore-size of 100 and $200 \mathrm{~nm}$ was purchased from Whatman (Whatman International Ltd.). The circle-shaped AAO membranes are in a diameter of $13 \mathrm{~mm}$ with a thickness of $60 \mu \mathrm{m}$. Highly purity $\mathrm{NO}$ (99.9\%) and $\mathrm{O}_{2}$ (99.7\%) were obtained from Zhonghong industry gas company (Shenzhen). Angeli's salt (AS) was purchased from Cayman chemical co. Stock solutions of AS was prepared in $10 \mathrm{mM}$ $\mathrm{NaOH}$ and stored at $-20^{\circ} \mathrm{C}$ for further use. All other regents were of analytical grade, and were used without further purification.

Standard saturated NO solution were prepared in the following procedure: firstly, oxygen-free deionized water was prepared by bubbling $\mathrm{N}_{2}$ gas for $30 \mathrm{~min}$. Then the deionized water bubbled with $\mathrm{NO}$ gas for $1 \mathrm{~h}$. Standard saturated $\mathrm{O}_{2}$ solution was also prepared in the similar way. The standard solutions were freshly prepared for each experiment and kept in a glass flask tightly with a rubber septum. The saturated concentration of $\mathrm{NO}$ and $\mathrm{O}_{2}$ in water at $25^{\circ} \mathrm{C}$ are $1.9 \mathrm{mM}$ and $0.263 \mathrm{mM}$, respectively. $50 \mathrm{mM}$ phosphate-buffered saline (PBS) containing $0.1 \mathrm{M} \mathrm{KCl}$ with $\mathrm{pH}$ value of 7.0 was used in this work.

\subsection{Preparation of nanofilm-Pt and NPt electrode}

The fabrication processes of nanofilm-Pt and NPt electrode are as follows: firstly, $\mathrm{N}$-type silicon wafer was rinsed with a standard "piranha" solution, a mixture of $3: 1(\mathrm{v} / \mathrm{v}) 98 \% \mathrm{H}_{2} \mathrm{SO}_{4}$ and $30 \% \mathrm{H}_{2} \mathrm{O}_{2}$. Meanwhile, AAO substrate was sonicated in anhydrous ethanol for $30 \mathrm{~min}$, rinsed with water, dried in air, and then fixed on a silicon plate. After the pretreatment, the cleaned silicon wafer, pore-size of 100 and $200 \mathrm{~nm}$ AAO substrates were used as templates for nanofilm-Pt, NPt-100 and NPt-200 electrodes, respectively. The Pt film were then fabricated by Nexdep Thin film 400 deposition system in base vacuum of approximately $1 \times 10^{-7}$ Torr. The deposition rate was set at $0.1 \AA / \mathrm{s}$. The thicknesses of Pt nanofilm on the three types of electrodes were around $40 \mathrm{~nm}$.

\subsection{Measurements}

Electrochemical measurements were performed at a $\mathrm{CHI} 660 \mathrm{E}$ electrochemical workstation (CHI Instruments, Inc. USA). A threeelectrode system was employed with $\mathrm{Ag} / \mathrm{AgCl}$ electrode as reference electrode, a platinum plate as counter electrode, and homemade nanofilm-Pt or NPt electrode (diameter, $3 \mathrm{~mm}$ ) as working electrode. All the potentials in this work were with respect to $\mathrm{Ag}$ / $\mathrm{AgCl}$ electrode and all measurements were carried out at room temperature $\left(25 \pm 2^{\circ} \mathrm{C}\right)$. EIS experimental was recorded using VersaSTAT 4 potentiostat/Galvanostat (Princeton Applied Research, AMETEK Inc, USA). The amplitude of modulation potential is $5 \mathrm{mV}$, and frequency range is from $10^{5}$ to $10^{-3} \mathrm{~Hz}$. All measurements were carried out at room temperature $\left(25 \pm 2{ }^{\circ} \mathrm{C}\right)$. Data analysis was performed with ZSimpWin 3.21 using the proposed equivalent circuit. Additionally, scanning electron microscopy (SEM) was obtained on a SU-70 (Hitachi) field emission scanning electron microanalysis at an operation potential of $5 \mathrm{kV}$. 


\section{Results and discussion}

\subsection{Morphological characterization}

As demonstrated in Fig. 1A and B, small nanoparticles could be observed in SEM images of nanofilm-Pt electrodes, which is due to many platinum nanoparticles had been deposited on silicon surface. In SEM images of nanoporous platinum electrodes (seen in Fig. 1C-F), platinum nanoparticles and holes are linked with each other regularly. Several structural features emerged from the micrographs. First, platinum film was deposited on AAO substrates mainly by surrounding the nanopores. Second, platinum film mainly consists of nanoparticles in good homogeneity. So, platinum nanoparticles link with each other along the AAO substrate frames to form a compact structure. The peculiar structure suggests that electric properties of as-fabricated nanoporous platinum electrode would be affected not only by that of individual particles but also by their linear and ring-shaped aggregates. The individual particles and their linear and ring shaped aggregates make the electrode possess larger active electrochemical area and higher conductivity, which facilitate the electron transfer and result in higher electrocatalytic activity of nanoporous platinum electrode. At the same time, the "nanocavities" observed in the nanoporous platinum
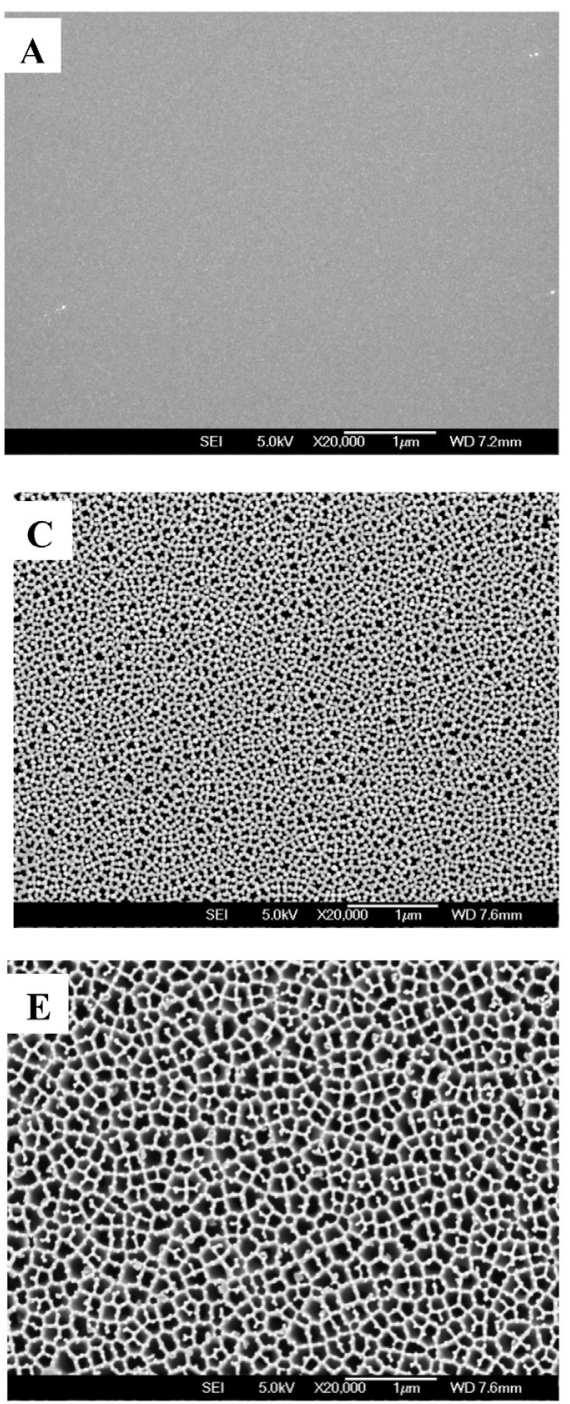

electrode are actually the 2-dimensional enclosures by linked nanoparticles and are different from the conventional arrays of "nanocavities" formed on a homogeneous metal film [20,21].

\subsection{CV characterization}

\subsection{1. $R u(N H)_{6}^{3+/ 2+}$}

Fig. 2 demonstrated $\mathrm{CVs}$ of $\mathrm{Ru}(\mathrm{NH})_{6}^{3+/ 2+}$ on nanofilm-Pt and NPt electrodes at different scan rates. At scan rate of $50 \mathrm{mV} / \mathrm{s}$, three pair of reversible redox peaks at $-0.177 \mathrm{~V},-0.188 \mathrm{~V}$, and $-0.210 \mathrm{~V}$ could be observed for nanofilm-Pt, NPt-100 and NPt-200 electrodes, respectively. Meanwhile, peak currents are linearly related to scan rates in the range from 5 to $50 \mathrm{mV} / \mathrm{s}$ on both nanofilm-Pt and NPt electrodes, which indicating a surface electron transfer process occurred [22]. Interestingly, after NPt electrodes undergone $\mathrm{CV}$ scanning in $\mathrm{Ru}(\mathrm{NH})_{6}^{3+/ 2+}$ solution, two pairs of broad redox peaks at $-0.221 \mathrm{~V}$ and $-0.225 \mathrm{~V}$ in blank buffer solution emerged for NPt-100 and NPt-200, respectively. It may due to $\mathrm{Ru}(\mathrm{NH})_{6}^{3+/ 2+}$ embedded in the nanopore of NPt electrodes after CV scanning processes. For control, only weak reduction peak around $-0.2 \mathrm{~V}$ was observed for nanofilm-Pt electrode in blank buffer solution (Fig. 2D), which is due to the different structure between NPt and nanofilm-Pt electrodes.
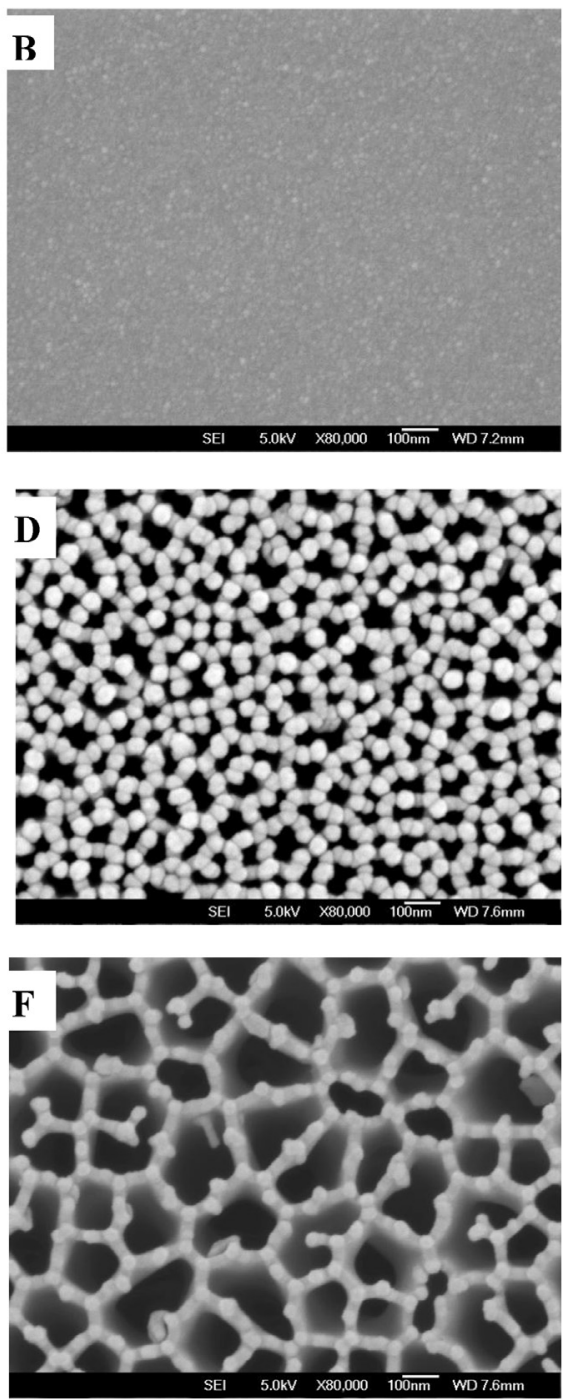

Fig. 1. Field-emission SEM images of nanofilm-Pt (A, B), NPt-100 (C, D) and NPt-200 (E, F) electrode surfaces. The magnitudes are $\times 20000$ and $\times 80000$, respectively. 

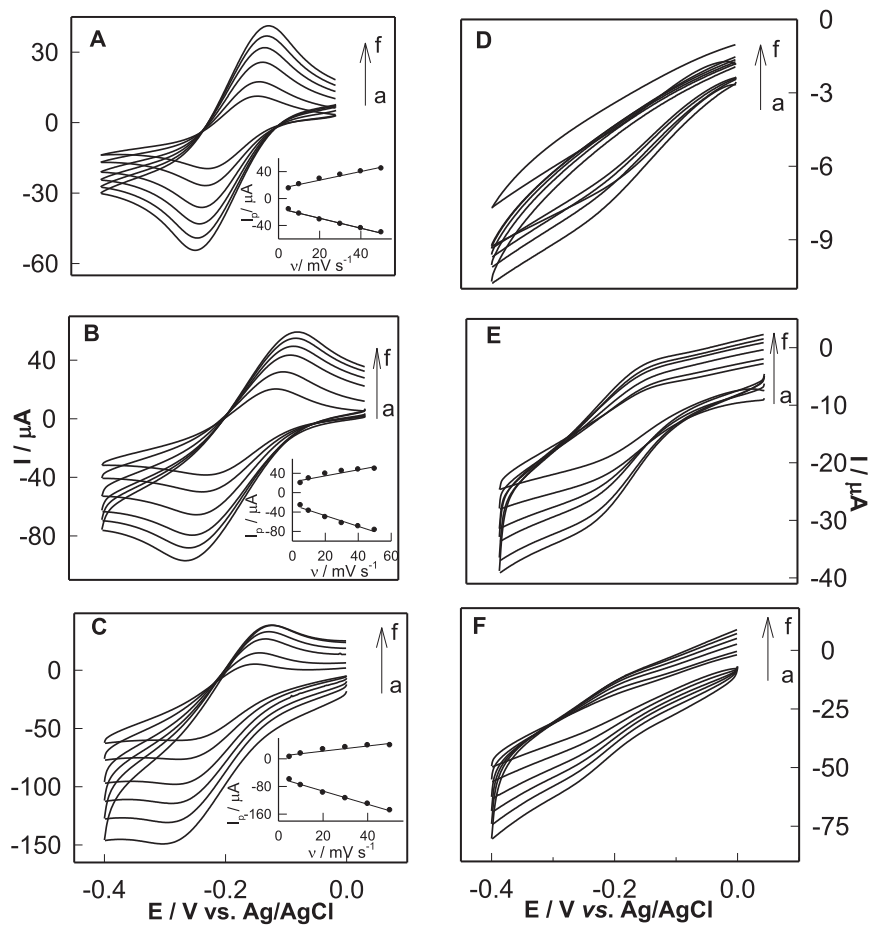

Fig. 2. CV responses of nanofilm-Pt (A), NPt-100 (B) and NPt-200 (C) electrodes in PBS buffer containing $5 \mathrm{mM} \mathrm{Ru}\left(\mathrm{NH}_{3}\right)_{6}^{3+/ 2+}$ at scan rate of (a) 5, (b) 10, (c) 20, (d) 30, (e) 40, (f) $50 \mathrm{mV} / \mathrm{s}$, respectively; the inset shows relationship between the peak current and scan rate; after CV scanning processes, CV responses of nanofilm-Pt (D), NPt-100 (E) and NPt-200 (F) electrodes in blank PBS buffer.

\subsection{2. $\mathrm{Fe}(\mathrm{CN})_{6}^{3-/ 4-}$}

Fig. 3 demonstrated $\mathrm{CVs}$ of $\mathrm{Fe}(\mathrm{CN}) 6^{3-/ 4-}$ on nanofilm-Pt and NPt electrodes at different scan rates. Compared with those on nanofilm-Pt electrode, the peak current increased near double and the formal potential shifted from $0.221 \mathrm{~V}$ to $0.234 \mathrm{~V}$ at scan rate of $50 \mathrm{mV}$ on NPt electrode. It suggests that NPt electrodes possess larger electro-active area. Meanwhile, as shown in the inset figures, peak currents are linear with the scan rate in the range from 5 to $50 \mathrm{mV} / \mathrm{s}$, which indicating a surface electron transfer process occurred.

\subsubsection{Calculation of electrochemical area}

Electrochemical areas of nanofilm-Pt and NPt electrodes can be determined by Randles-Sevcik equation for a reversible redox couple [23]:

$I_{p}=\left(2.69 \times 10^{5}\right) n^{3 / 2} A D^{1 / 2} v^{1 / 2} C_{0}$

where $I_{\mathrm{p}}$ is peak current (A), $n$ is electron transfer number, $A$ is electrode area $\left(\mathrm{cm}^{2}\right), D$ is diffusion coefficient of electro-active species $\left(\mathrm{cm}^{2} / \mathrm{s}\right), C_{0}$ is bulk concentration of the same species $\left(\mathrm{mol} / \mathrm{cm}^{3}\right)$, and $v$ is scan rate $(\mathrm{V} / \mathrm{s})$. If a reversible electro-active species is used with a known diffusion coefficient, electrode area can be calculated from peak current of a cyclic voltammogram. The diffusion coefficient of $5 \mathrm{mM} \mathrm{K}_{3} \mathrm{Fe}(\mathrm{CN})_{6}$ in a PBS solution is $1 \times 10^{-5} \mathrm{~cm}^{2} / \mathrm{s}$ at $25^{\circ} \mathrm{C}$ [24]. Therefore, electrochemical areas of nanofilm-Pt and NPt electrodes can be obtained from CV data (shown in Fig. 3). Results are listed in Table 1. Obviously, geometric area of nanofilm-Pt electrode is larger than that of NPt electrode, due to the porous structure of NPt electrode. However, electrochemical area of NPt electrode is nearly-double larger than that of nanofilm-Pt electrode. It means that electrochemical probe

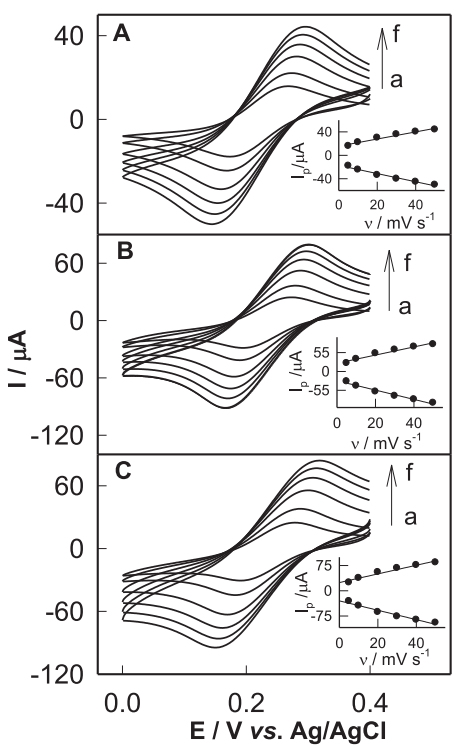

Fig. 3. CV responses of nanofilm-Pt (A), NPt-100 (B) and NPt-200 (C) electrodes in PBS buffer containing $5 \mathrm{mM} \mathrm{Fe}(\mathrm{CN})_{6}^{3-/ 4-}$ at scan rate of (a) 5 , (b) 10 , (c) 20, (d) 30, (e) 40, (f) $50 \mathrm{mV} / \mathrm{s}$, respectively; the inset shows relationship between the peak current and scan rate.

produces larger $\mathrm{CV}$ response on NPt electrode than that on nanofilm electrode. Possible reason is as follows: owing to the presence of one pore between the two patch of nanoparticle, the diffusion mode of electrochemical probe is global diffusion on the NPt electrode. While on the nanofilm electrode, the diffusion mode is planar diffusion.

\subsection{EIS characterization}

Concerning the special structure of nanofilm and nanoporous platinum electrode, present Randles model was not applicable $[25,26]$. Two new equivalent circuit models with paralleled (RQ) or (RT) component was proposed for nanofilm-Pt and NPt electrodes in this work, shown in Fig. 4. Electronic elements in new equivalent circuits are described below. Solution resistance $\left(R_{S}\right)$, is related to the resistance between reference electrode and working electrode. $\mathrm{R}_{\mathrm{ct}}$ is the electron-transfer resistance; $C_{\mathrm{dl}}$ accounts for double layer capacitance; $\mathrm{Q}$ acts as a nonlinear capacitor related to film in nanopore and electrode surface; $\mathrm{W}$ is the Warburg diffusion resistance; $T$ is the impedance caused by global diffusion.

Fig. 5 illustrates the Nyquist plots of nanofilm-Pt, NPt-100 and NPt-200 electrodes in $5 \mathrm{mM} \mathrm{Fe}(\mathrm{CN})_{6}^{3-/ 4-}$ solution. Fitted data was also shown as solid points. The impedance spectra include a semicircle and linear portion. The semicircle portion at higher frequency range corresponds to electron-transfer-limited process, and linear portion at lower frequency range represents the

Table 1

Comparison of the geometric and electrochemical areas on the nanofilm-Pt electrode with those on the NPt electrode.

\begin{tabular}{lll}
\hline Electrodes & Geometric area $\left(\mathrm{mm}^{2}\right)$ & Electrochemical area ${ }^{\mathrm{b}}\left(\mathrm{mm}^{2}\right)$ \\
\hline Nanofilm-Pt & $7.05 \pm 0.06$ & $5.96 \pm 0.08$ \\
NPt-100 & $3.82 \pm 0.04$ & $8.95 \pm 0.09$ \\
NPt-200 & $2.16 \pm 0.03$ & $9.38 \pm 0.11$ \\
\hline
\end{tabular}

a The geometric area are calculated from the following equation: $A=\pi r^{2}$ $(\mathrm{r}=1.5 \mathrm{~mm})$, for NPt-100 and NPt-200 electrodes, the filling factor are 0.5433 and 0.3084 , respectively.

b Calculated from equation (3). 


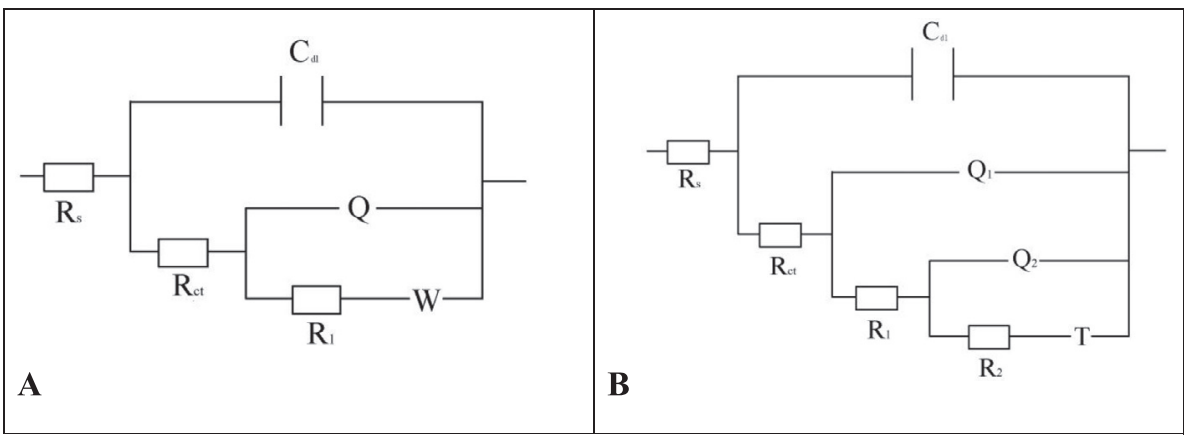

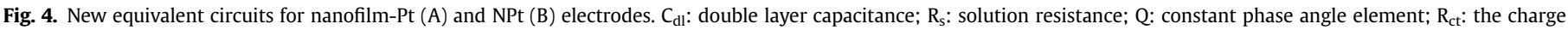
transfer resistance; $R_{1}$ : resistance through the nanoparticles; $R_{2}$ : resistance through the nanoholes; $T$ : finite length diffusion, blocking at a surface (Tanhyperbol).

diffusion-limited process. Obviously, an semicircle portion with a straight line for the spectrum of $\mathrm{Fe}(\mathrm{CN})_{6}^{3-14-}$ can be observed on nanofilm-Pt electrode (Fig. 5A). While the spectrum of $\mathrm{Fe}(\mathrm{CN})_{6}^{3-/ 4-}$ on NPt electrodes shows two quasi-straight lines in high frequency region and low frequency region, respectively (Fig. 5B and C). Compared with that on nanofilm-Pt electrode, there are lines with different slopes on NPt electrodes. It indicates different diffusionlimited processes of $\mathrm{Fe}(\mathrm{CN})_{6}^{3-/ 4-}$ on NPt electrode surface. Meanwhile, compared with that on nanofilm-Pt electrode $\left(14.05 \Omega \cdot \mathrm{cm}^{2}\right)$, the impedance spectrum on NPt electrodes exhibited a semicircle portion with much smaller diameters $\left(1.24 \Omega \cdot \mathrm{cm}^{2}\right.$ for NPt-100, 1.48 $\Omega \cdot \mathrm{cm}^{2}$ for NPt-200). It means that the electron-transfer rate increases rapidly on NPt electrodes.

As demonstrated in Fig. 5, the EIS data was fitted by proposed equivalent circuit effectively. Results are listed in Table 2. $\mathrm{C}_{\mathrm{dl}}$ accounts for double layer capacitance, which depends on many variables including electrode potential, temperature, ionic

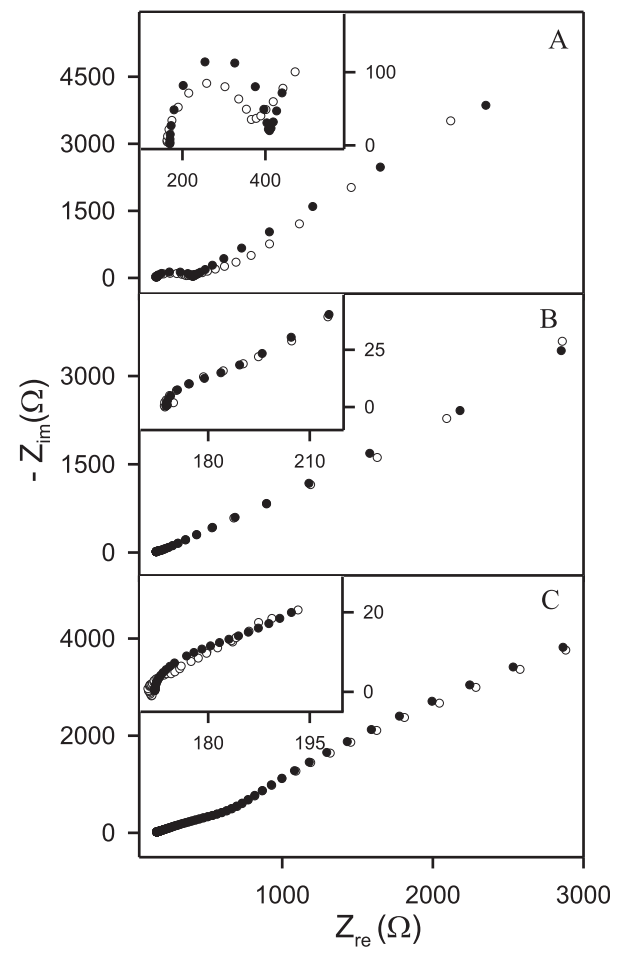

Fig. 5. Representative Nyquist plots of nanofilm-Pt (A), NPt-100 (B) and NPt-200 (C) electrodes in PBS buffer containing $5 \mathrm{mM} \mathrm{Fe}(\mathrm{CN})_{6}^{3-/ 4-}$. $\bigcirc$ : experimental data; $\bullet$ : fitting data, calculated by the new equivalent circuit concentrations, oxide layers, and electrode roughness etc. These variables are the same except electrode roughness between the nanofilm Pt and NPt electrodes. The larger the electrode roughness, the thicker the diffusion layer. Therefore, the larger the double layer capacitance. Owing to the nanopore structure, the electrode roughness of NPt electrode is larger than that of nanofilm Pt electrode. Therefore, $\mathrm{C}_{\mathrm{dl}}$ on NPt electrode ( 155 and $340 \mu \mathrm{F} / \mathrm{cm}^{2}$ ) is larger than that of nanofilm Pt electrode $\left(24.3 \mu \mathrm{F} / \mathrm{cm}^{2}\right)$. The Warburg diffusion resistance (W) was incorporated in equivalent circuit model due to planar diffusion occurred on nanofilm electrode. Meanwhile, $\mathrm{T}$ impedance was incorporated in equivalent circuit model due to global diffusion occurred on NPt electrode. Since there are two different diffusion types on the two electrodes, no comparability of the $\mathrm{W}$ and $\mathrm{T}$ value for nanofilm and NPt electrodes.

\subsection{Electrocatalytic activity of $\mathrm{NPt}$ electrode toward $\mathrm{O}_{2}, \mathrm{NO}, \mathrm{AS}$}

Since NPt-200 electrode possesses a little larger electrochemical area than that of NPt-100 electrode, the former was adopted for electrocatalytic activity study in the following experimental. Under normal condition $\left(101.325 \mathrm{KPa}, 25^{\circ} \mathrm{C}\right)$, the concentration of saturated $\mathrm{O}_{2}$ in water is $0.263 \mathrm{mM}$. Fig. 6 shows $\mathrm{CV}$ responses of nanofilm-Pt and NPt-200 electrode in the presence of saturated $\mathrm{O}_{2}$. The electrocatalytic reduction potential occurred at $-0.012 \mathrm{~V}$ for nanofilm-Pt electrode and $0.094 \mathrm{~V}$ for NPt-200 electrode, respectively. The positive shift in potential is $105.8 \mathrm{mV}$, which indicating NPt electrode possesses higher electrocatalytic activity for. Meanwhile, the catalytic sensitivity of NPt-200 electrode to $\mathrm{O}_{2}$ is $0.85 \mathrm{~mA} \mathrm{~cm}^{-2}$, which is larger than that of nanofilm-Pt electrode $\left(0.54 \mathrm{~mA} \mathrm{~cm}^{-2}\right)$.

Fig. 7 demonstrated the catalytic responses of nanofilm-Pt and NPt-200 electrode to NO in buffers with different pH values. For

Table 2

Comparison of fitted data calculated by the respective equivalent circuit model for nanofilm-Pt and NPt electrodes.

\begin{tabular}{llll}
\hline Parameters & Nanofilm-Pt & NPt-100 & NPt-200 \\
\hline $\mathrm{R}_{\mathrm{s}}\left(\Omega \cdot \mathrm{cm}^{2}\right)$ & 10.24 & 15.06 & 16.20 \\
$\mathrm{C}_{\mathrm{dl}}\left(\mu \mathrm{F} / \mathrm{cm}^{2}\right)$ & 24.3 & 155 & 340 \\
$\mathrm{R}_{\mathrm{ct}}\left(\Omega \cdot \mathrm{cm}^{2}\right)$ & 14.05 & 1.24 & 1.48 \\
$\mathrm{Q}_{1}-\mathrm{Y}_{0}$ & 0.0033 & 0.0016 & 0.0016 \\
$\mathrm{Q}_{1}-\mathrm{n}$ & 0.70 & 0.53 & 0.58 \\
$\mathrm{R}_{1}\left(\Omega \cdot \mathrm{cm}^{2}\right)$ & 3.45 & 44.13 & 160.17 \\
$\mathrm{Q}_{2}-\mathrm{Y}_{0}$ & $N A$ & 0.00015 & 0.0021 \\
$\mathrm{Q}_{2}-\mathrm{n}$ & $N A$ & 0.78 & 0.93 \\
$\mathrm{R}_{2}\left(\Omega \cdot \mathrm{cm}^{2}\right)$ & $N A$ & 490 & 529 \\
$\mathrm{~T}(\mathrm{~W})-\mathrm{Y}_{0}$ & $1.37 \times 10^{6}$ & $1 \times 10^{6}$ & $7.25 \times 10^{6}$ \\
$\mathrm{~T}-\mathrm{B}$ & $N A$ & 4.06 & 59.29 \\
\hline
\end{tabular}

NA: not applicable. 


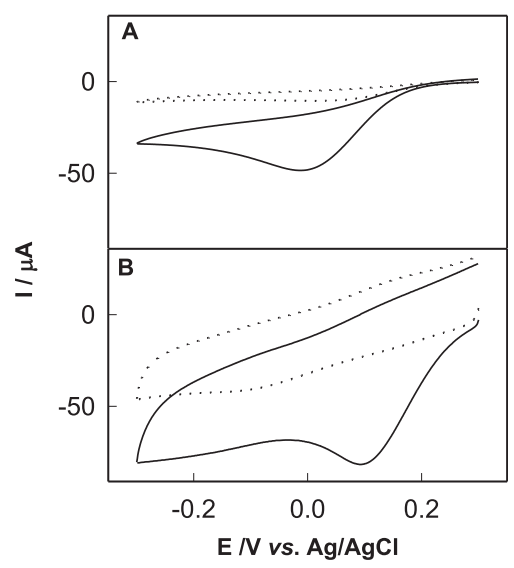

Fig. 6. CVs of nanofilm-Pt (A) and NPt-200 (B) electrodes in the absence (dotted line) and presence (solid line) of saturated $\mathrm{O}_{2}(0.263 \mathrm{mM})$, respectively. Condition: $50 \mathrm{mM}$ PBS buffer solution ( $\mathrm{pH} 7.4$ ); scan rate: $50 \mathrm{mV} / \mathrm{s}$; number of observation was $n=3$.

nanofilm-Pt electrode, reduction peaks were observed at $-0.40 \mathrm{~V}$ in $\mathrm{pH} 5.4$ solution, $-0.75 \mathrm{~V}$ in $\mathrm{pH} 7.4$ and $\mathrm{pH} 9.4$ solutions, respectively. Meanwhile, oxidation current was observed in $\mathrm{pH} 9.4$ solution although no peak emerged. While no oxidation current was observed in $\mathrm{pH} 5.4$ and 7.4 solution. For NPt-200 electrode, reduction peaks were observed at $-0.52 \mathrm{~V}$ in $\mathrm{pH} 5.4$ solution, $0.68 \mathrm{~V}$ in $\mathrm{pH} 7.4$ and $-0.60 \mathrm{~V}$ in $\mathrm{pH} 9.4$ solutions. Meanwhile, oxidation peak current was observed at $0.90 \mathrm{~V}$ in $\mathrm{pH} 5.4$ solution, $0.84 \mathrm{~V}$ in $\mathrm{pH} 7.4$ and $0.95 \mathrm{~V}$ in $\mathrm{pH} 9.4$ solutions. At all the three $\mathrm{pH}$ values (5.4, 7.4 and 9.4) of buffer solutions, NPt-200 electrode exhibited almost ten times larger catalytic current both in the oxidation and reduction potential range than those for nanofilm-Pt electrode. It indicates that the NPt electrode exhibits better catalytic activity to NO than that of nanofilm-Pt electrode. Meanwhile, the catalytic current increases with the increase of $\mathrm{pH}$ value, which indicating that alkaline condition is beneficial to NO electrocatalysis.

Fig. 8 demonstrates $\mathrm{CV}$ responses of AS in different $\mathrm{pH}$ solution on nanofilm-Pt and NPt-200 electrodes. Generally, oxidation and
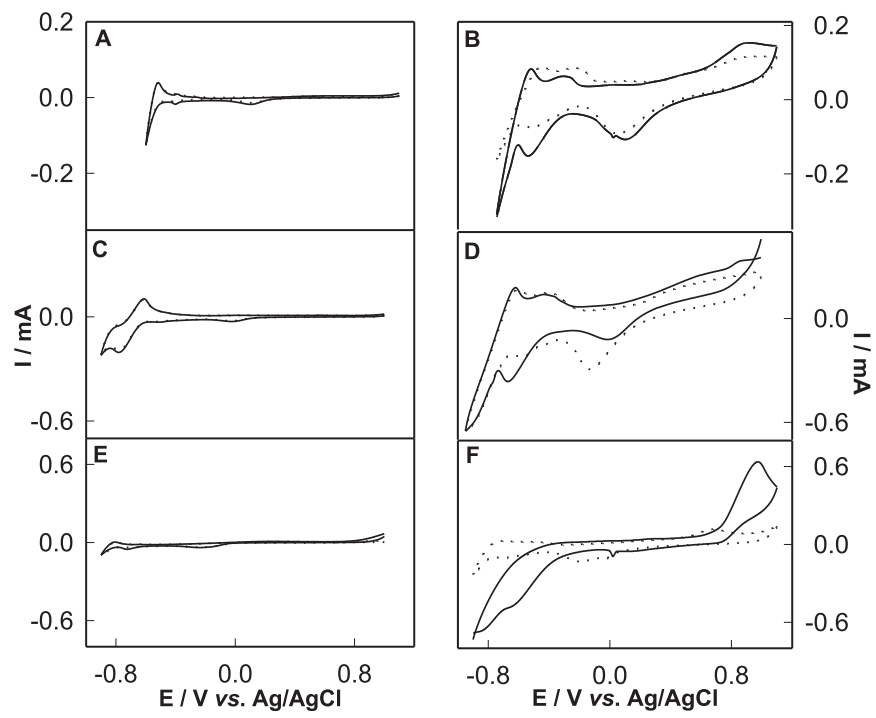

Fig. 7. CVs of nanofilm-Pt (A, C, E) and NPt-200 (B, D, F) electrodes in the absence (dotted line) and presence (solid line) of $50 \mathrm{mM}$ PBS buffer containing $2 \mu \mathrm{M} \mathrm{NO}$ respectively. Condition: 50 mM PBS buffer pH 5.4 (A,B), pH 7.4 (C, D) and pH 9.4 (E, F); scan rate: $50 \mathrm{mV} / \mathrm{s}$ reduction peaks emerged on both nanofilm-Pt and NPt-200 electrodes in all the three $\mathrm{pH}$ value (5.4, 7.4 and 9.4) of buffer solutions except that on nanofilm-Pt electrode in $\mathrm{pH}$ value of 9.4. It may be due to more stability of AS in $\mathrm{pH} 9.4$ solution and weaker catalyticactivity of nanofilm-Pt electrode. Comparatively, larger catalytic current was observed on NPt-200 electrode in the same $\mathrm{pH}$ value solution, and larger catalytic current was obtained in the $\mathrm{pH}$ value of 5.4 at the same electrode. The reason is as follows: first, due to its frame and porous structure, NPt-200 electrode possesses larger electro-active area than that of nanofilm-Pt electrode; second, among the three $\mathrm{pH}$ value of buffer solutions, the decomposing rate of AS to HNO is fastest in $\mathrm{pH} 5.4$.

Fig. 9 shows CV responses of nanofilm-Pt and NPt-200 electrodes to different concentration of AS in pH 7.4 buffer. Obviously, an increase in anodic peak current and a decrease in cathodic peak current were observed after the addition of AS. Meanwhile, the response currents are linear with the AS concentrations. The increase for NPt-200 electrode was larger than that for nanofilm-Pt electrode at the same concentration of AS. Obviously, with the increase of AS concentration, the oxidation potential shifts positively and the reduction potential shifts negatively.

\subsection{Electrocatalytic mechanism of NPt electrode toward NO and HNO}

The electrocatalytic reduction mechanisms of NPt electrode toward NO and HNO are as follows, taking NO as an example:

When solution $\mathrm{pH} \gg 7$,

$\mathrm{Pt}^{*}+\mathrm{NO}_{(\text {sol })} \rightarrow \mathrm{Pt}-\mathrm{NO}_{(\mathrm{ads})}$

$\mathrm{Pt}-\mathrm{NO}_{(\mathrm{ads})}+2 \mathrm{H}^{+}+2 \mathrm{e}^{-} \rightarrow \frac{1}{2} \mathrm{~N}_{2}+\mathrm{H}_{2} \mathrm{O}+\mathrm{Pt}^{*}$

When solution $\mathrm{pH} \ll 7$,

$\mathrm{Pt}^{*}+\mathrm{H}^{+}(\mathrm{sol})+\mathrm{e}^{-} \rightarrow \mathrm{Pt}-\mathrm{H}_{(\mathrm{ads})}$

$2 \mathrm{Pt}-\mathrm{H}_{(\mathrm{ads})}+\mathrm{NO}+\mathrm{e}^{-} \rightarrow 2 \mathrm{Pt}^{*}+\mathrm{H}_{2} \mathrm{O}+\frac{1}{2} \mathrm{~N}_{2}$
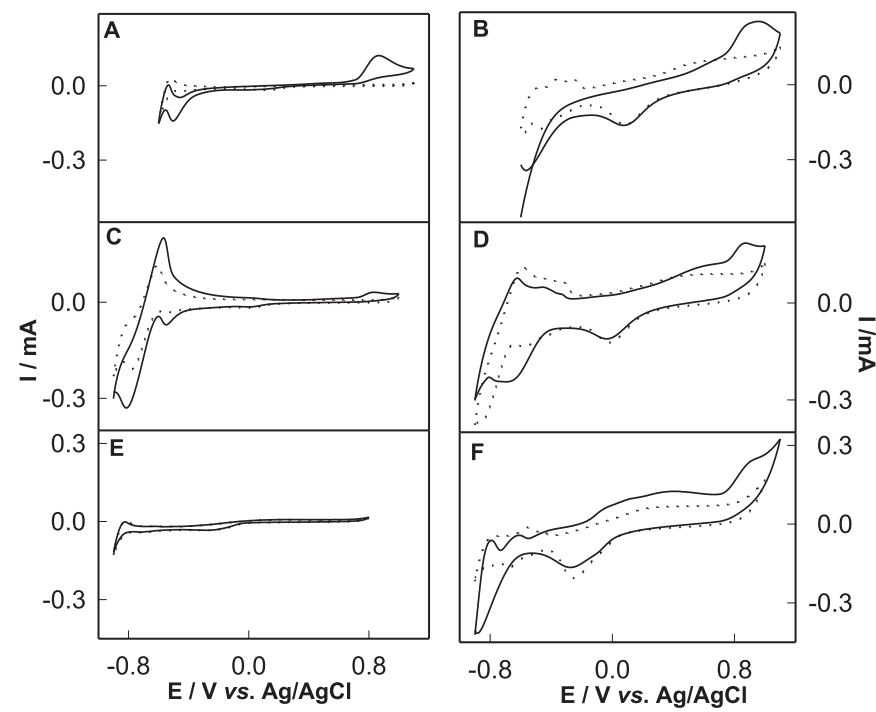

Fig. 8. CVs of nanofilm-Pt (A, C, E) and NPt-200 (B, D, F) electrodes in the absence (dotted line) and presence (solid line) of $50 \mathrm{mM}$ PBS buffer containing $0.1 \mathrm{mM}$ AS, respectively. Condition: 50 mM PBS buffer pH 5.4 (A,B), pH 7.4 (C,D) and pH 9.4 (E, F); scan rate: $50 \mathrm{mV} / \mathrm{s}$ 


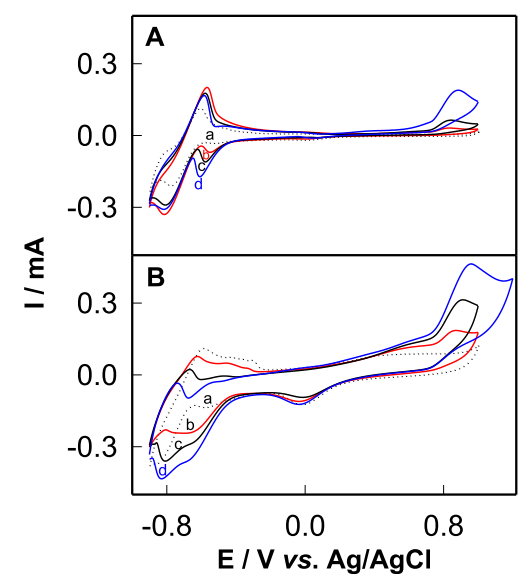

Fig. 9. CVs of nanofilm-Pt (A) and NPt-200 (B) electrodes in the absence (a), and presence of $0.1 \mathrm{mM}$ (b), $0.2 \mathrm{mM}$ (c) and $0.3 \mathrm{mM}$ (d) AS, respectively. Condition: $50 \mathrm{mM}$ PBS buffer, $\mathrm{pH} 7.4$; scan rate: $50 \mathrm{mV} / \mathrm{s}$.

The electrocatalytic oxidation mechanisms of NPt electrode toward NO and HNO are as follows, also taking NO as an example:

$\mathrm{Pt}^{*}-2 \mathrm{e}^{-} \rightarrow \mathrm{Pt}^{2+}$ (fast)

$\mathrm{Pt}^{2+}+\mathrm{NO}_{(\text {sol })} \rightarrow \mathrm{Pt}^{2+}-\mathrm{NO}_{(\text {ads })}$

$\mathrm{Pt}^{2+}-\mathrm{NO}_{(\mathrm{ads})}+2 \mathrm{H}_{2} \mathrm{O}-3 \mathrm{e}^{-} \rightarrow \mathrm{NO}_{3}^{-}+4 \mathrm{H}^{+}+\mathrm{Pt}^{2+*}$

Then toward cathodic potential scan, the reduction of Pt oxide layer occurred:

$\mathrm{Pt}^{2+*}+2 \mathrm{e} \rightarrow \mathrm{Pt}^{*}$

\section{Conclusions}

In this work, a new type of nanoporous platinum (NPt) electrode was successfully prepared on a porous aluminum oxide membrane by thermal evaporation deposition technology. The morphology study showed that the stereo-structure of "nanocavities" on NPt electrode make it possesses larger specific surface area. EIS data indicated that NPt electrode possesses higher conductivity. The electrocatalytic studies indicated that NPt electrode exhibited higher electrocatalytic activity to reactive oxygen $\left(\mathrm{O}_{2}\right)$ and nitrogen species (NO and Angeli's salt) than those of nanofilm electrode. In electrocatalytic reduction of $\mathrm{O}_{2}$, the positive shift in reduction potential was $105.8 \mathrm{mV}$, which indicating NPt electrode possesses higher electrocatalytic activity. In both the oxidation and reduction potential range, NPt-200 electrode exhibited almost ten times and three times larger catalytic current than those for nanofilm-Pt electrode toward electrocatalysis of NO and AS, respectively. Electrocatalytic oxidation and reduction mechanisms toward NO and AS depend on $\mathrm{pH}$ value of buffer solution.

\section{Acknowledgment}

This work was supported by the National Natural Science Foundations of China (No. 21405106), the Youth Innovation Promotion
Association of Chinese Academy of Sciences (2011170), the Research and Development Foundation of Science and Technology of Shenzhen (Nos. JCYJ20150324141711592 and 20180502154158198).

\section{References}

[1] E. Skotadis, G. Tsekenis, M. Chatzipetrou, L. Patsiouras, L. Madianos, Heavy metal ion detection using DNAzyme-modified platinum nanoparticle networks, Sens. Actuators, B 239 (2017) 962.

[2] L. Liu, B. Du, C. Shang, J. Wang, E. Wang, Construction of surface chargecontrolled reduced graphene oxide-loaded Fe3O4 and Pt nanohybrid for peroxidase mimic with enhanced catalytic activity, Anal. Chim. Acta 1014 (2018) 77.

[3] R.M. Antoniassi, L. Otubo, J.M. Vaz, A.O. Neto, E.V. Spinace, Synthesis of Pt nanoparticles with preferential (100) orientation directly on the carbon support for direct ethanol fuel cell, J. Catal. 342 (2016) 67.

[4] M. Cueto, M. Piedrahita, C. Caro, B. Martínezhaya, M. Sanz, Platinum nanoparticles as photoactive substrates for mass spectrometry and spectroscopy sensors, J. Phys. Chem. C118 (2014) 11432.

[5] G.E. Thompson, Porous anodic alumina: fabrication, characterization and applications, Thin Solid Films 297 (1997) 192.

[6] C.J. Li, Y.G. Guo, B.S. Li, C.R. Wang, L.J. Wan, C.L. Bai, Template synthesis of Sc@ C82(I) nanowires and nanotubes at room temperature, Adv. Mater. 17 (2005) 71.

[7] Y. Park, S. Kim, I.H. Jang, Y.S. Nam, H. Hong, D. Choi, W.G. Lee, Role of the electric field in selective ion filtration in nanostructures, Analyst 1 (2016) 451.

[8] F. Liu, J.Y. Lee, W.J. Zhou, Segmented Pt/Ru, Pt/Ni, and Pt/RuNinanorods as model bifunctional catalysts for methanol oxidation, Small 2 (2006) 121.

[9] A. Santos, T. Kumeria, D. Losic, Nanoporous anodic aluminum oxide for chemical sensing and biosensors, Trends Anal. Chem. 44 (2013) 25.

[10] P. Ciambelli, L. Arurault, M. Sarno, S. Fontorbes, C. Leone, L. Datas, D. Sannino, P. Lenormand, S. Le Blond Du Plouy, Controlled growth of CNT in mesoporous AAO through optimized conditions for membrane preparation and CVD operation, Nanotechnology 22 (2011), 265613.

[11] C.C. Lu, Y.S. Huang, J.W. Huang, C.K. Chang, S.P. Wu, A macroporous TiO2 oxygen sensor fabricated using anodic aluminumoxide as an etching mask, Sensors 10 (2010) 670.

[12] H. Masuda, K. Fukuda, Fabrication of highly ordered structures using anodic porous alumina, Science 268 (1995) 1466.

[13] H. Shimokawa, S. Godo, Diverse functions of endothelial NO synthases system: NO and EDH, J. Cardiovasc. Pharmacol. 67 (2016) 361.

[14] F. Oladayo, K.A. Mills, D.J. Sellers, C.W. Russ, Three gaseous neurotransmitters, nitric oxide, carbon monoxide, and hydrogen sulfide, are involved in the neurogenic relaxation responses of the porcine internal anal sphincter, J. Neurogastroent. MotiL 22 (2016) 141.

[15] Z. Zhang, C.J. Smith, W. Li, J. Ashworth, Characterization of nitric oxide modulatory activities of alkaline-extracted and enzymatic-modified arabinoxylans from corn bran in cultured human monocytes, J. Agric. Food Chem. 43 (2016) 64.

[16] Q. Cheng, Y.X. Jiang, N. Tian, Z.Y. Zhou, S.G. Sun, Electrocatalytic reduction of nitric oxide on Pt nanocrystals of different shape in sulfuric acid solutions, Electrochim. Acta 55 (2010) 8273.

[17] M. Cetraz, V. Sen, S. Schoch, K. Streule, V. Golubev, A. Hartwig, B. Köberle, Platinum(IV)-nitroxyl complexes as possible candidates to circumvent cisplatin resistance in RT112 bladder cancer cells, Arch. Toxicol. 91 (2017) 785.

[18] C.L. Bianco, J.P. Toscano, M.D. Bartberger, J.M. Fukuto, The chemical biology of HNO signaling, Arch. Biochem. Biophys. 617 (2016) 129.

[19] F. Doctorovich, D.E. Bikiel, J. Pellegrino, S.A. Suarez, M.A. Marti, Reactions of HNO with metal porphyrins: underscoring the biological relevance of HNO, Acc. Chem. Res. 47 (2014) 2907.

[20] A.G. Brolo, E. Arctander, R. Gordon, B. Leathem, K.L. Kavanagh, Nanoholeenhanced Raman scattering Nano Lett. 4 (2004) 2015.

[21] J.V. Coe, K.R. Rodriguez, S. Teeters-Kennedt, K. Cilwa, J. Heer, H. Tian, S.M. Williams, Metal films with arrays of tiny holes: spectroscopy with infrared plasmonicscaffolding, J. Phys. Chem. C 111 (2007) 17459.

[22] H.Y. Gu, A.M. Yu, H.Y. Chen, Direct electron transfer and characterization of hemoglobin immobilized on an Au colloid-cysteamine-modified gold electrode, J. Electroanal. Chem. 516 (2001) 119.

[23] A.J. Bard, L.R. Faulkner, Electrochemical Methods, John Wiley and Sons, Chichester, 1980.

[24] J.J. Gooding, V.G. Praig, E.A.H. Hall, A. Chem, Platinum-catalyzed enzyme electrodes immobilized on gold using self-assembled layers, Anal. Chem. 70 (1998) 2396.

[25] Y.T. Long, C.Z. Li, H.B. Kraatz, J.S. Lee, AC impedance spectroscopy of native DNA and M-DNA, Biophys. J. 84 (2003) 3218.

[26] X. Li, J.S. Lee, H.B. Kraatz, Electrochemical detection of single-nucleotide mismatches using an electrode microarray, Anal. Chem. 78 (2006) 6096. 